\title{
Time Delay Estimation for Passive Sonar Signal Processing
}

\author{
G. CLIFFORD CARTER, SENIOR MEMBER, IEEE
}

\begin{abstract}
An overview of applied research in passive sonar signal processing estimation techniques for naval systems is presented. The naval problem that motivates time delay estimation is the source state estimation problem. A discussion of this problem in terms of estimating the position and velocity of a moving acoustic source is presented. Optimum bearing and range estimators are presented for the planar problem and related to the optimum time delay vector estimator. Suboptimum realizations are considered together with the effects of source motion and receiver positional uncertainty.
\end{abstract}

\section{INTRODUCTION}

$\mathrm{T}$ HE purpose of this paper is to provide a tutorial review of time delay estimation in the passive sonar signal processing field.

In the passive sonar problem of interest here, signals received at two or more receiving sensors or hydrophones are used to estimate the position and velocity of a detected acoustic source. Passive systems, unlike radar or active sonar systems, cannot control the amount of transmitted energy to be reflected off the source; however, the covertness of passive systems can be advantageous, both in military and biomedical applications. A discussion of radar and active sonar can be found in Altes [3]. In practice, the number of receiving sensors, the observation time and the ratio of the background noise to the source signal strength after propagation loss, when balanced against total system cost, dictate the feasibility of passive systems.

In the ocean, sound usually arrives at each individual omnidirectional receiver through more than one path. For example, sound may arrive through the direct and surface-reflected paths, as shown in Fig. 1. A more complete discussion of recent research in underwater acoustics is available in the texts edited by Oppenheim [44] and Bj申rn $\phi$ [7]. To make the problem mathematically tractable from a signal processing point of view, it is convenient to decouple the problem into multipath and planar problems. For multipath signals, a simplistic model of the received signal is that each receiver sees a signal plus an attenuated and delayed signal corrupted by additive uncorrelated

Manuscript received September 26, 1980; revised November 20, 1980. This work summarizes research completed at the University of Connecticut, portions of which were presented at the 1976 North Atlantic Treaty Organization (NATO) Advanced Study Institute (ASI) on Underwater Acoustics and Signal Processing (UASP) in Portovenere, Italy. It also summarizes work completed since that time by the author and others working in this field. Portions of this work were also presented at IEEE EASCON '79, Washington, DC, at the 13th Annual Asilomar Conference on Circuits, Systems, and Computers, Monterey, CA, and at the 1980 NATO ASI on UASP, Kollekolle, Denmark.

The author is with the Naval Underwater Systems Center, New London, CT 06320.

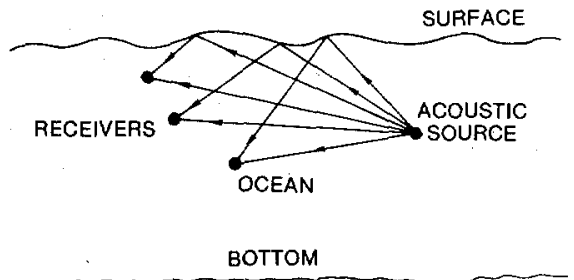

Fig. 1. Model of direct and surface-reflected sound ray paths received at three sensors.

noise. For the planar problem (i.e., when all the receivers and the source are in the same plane), the time delay to be estimated is the travel time of the acoustic wavefront between pairs of receivers, so that the source position and velocity can be estimated. The type of signal processing to be used and the bounds on performance for such processing are important subjects of this paper.

\section{The Mathematical Model}

For the purpose of this paper, only the planar problem will be considered. In particular, it will be assumed that acoustic energy arrives at each receiver through only one propagation path in the same plane with all the receivers and source.

Pictorially, we are interested in the delay of the signal from one receiving sensor to the next, as shown in Fig. 2. For zero relative time delay, the source bearing is broadside to the sensor pair; and for maximum time delay, the source bearing is endfire. A certain amount of caution must be exercised in applying the results directly to "real world" problems, since the ocean medium is more complex than this simple model. More sophisticated propagation modeling and associated signal processing is considered by Owsley [45]. However, considerable insight can be gained in dealing with the passive bearing estimation problem in a simplified decoupled fashion.

A simple mathematical model for the received signals in the planar case is

$$
\begin{aligned}
& r_{1}(t)=s(t)+n_{1}(t) \\
& r_{2}(t)=s(t-D)+n_{2}(t)
\end{aligned}
$$

where the source signal $s(t)$ and the noises $n_{1}(t)$ and $n_{2}(t)$ are Gaussian, stationary, and mutually uncorrelated. After the signal has been detected, an important passive sonar problem is to estimate the time delay $D$ between two sensors separated by length $L$. This time delay estimate then is used to estimate the bearing angle shown in Fig. 2. The bearing estimate is given by the approximate rule

$$
\hat{B} \cong \cos ^{-1}(C \hat{D} / L)
$$




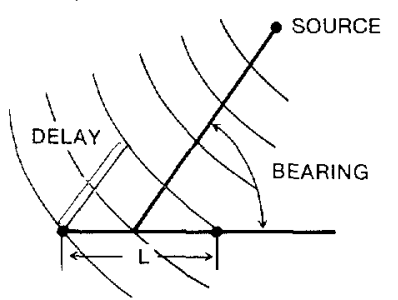

Fig. 2. Planar model of two receivers separated by a distance $L$.

where

1) $C$ (without a frequency argument) is the speed of sound in water;

2) $\hat{B}$ is the bearing estimate;

3) $\hat{D}$ is the time delay estimate. It can be shown that $B$ is the angle that the hyperbolic "line of position" makes with the axis of the receivers; hence the approximation equation (2) is increasingly accurate as the range to the acoustic source increases.

\section{System Configurations FOR Time Delay ESTIMATION}

The critical part of the passive bearing estimation problem is the accurate estimation of time delay. Two conceptual configurations come to mind based on either 1) an intuitive approach and a familiarity with detection theory, or 2) a rigorous application of the maximum likelihood (ML) approach for white signals in white noise. In both conceptual configurations we attempt to "advance". the delayed received signal by a hy. pothesized amount in order to align it with the other received signal. Then we either sum, square, and average, as shown in Fig. 3, or we multiply and average as shown in Fig. 4. In both cases the hypothesized delays are adjusted in order to maximize the configuration output. As shown in the figures, both configuration outputs consist of "signal-cross-signal" terms. Further, both configurations are ML estimators for time delay under the assumptions that the signal and noises are white and mutually uncorrelated. A further discussion of the cross correlator configuration for time delay estimation is given by Bendat and Piersol [6]. When the signal and noise spectral characteristics are nonwhite, the received waveforms must be prefiltered with particular equiphase filters (i.e., the prefilters must have the same phase characteristics) to accentuate the frequency bands with good signal-to-noise ratio (SNR). It is of interest to note that a signal detector can be realized by comparing either configuration output to a threshold. Moreover, in terms of detection (but not estimation) in the presence of noise, the system in Fig. 3 outperforms the system in Fig. 4; however, the system in Fig. 3 requires prior knowledge of power levels in order to set the proper threshold. The system in Fig. 4 has a zero mean output in the signal absent, noise present case.

The conceptual systems in Figs. 3 and 4 can be achieved in a number of different ways. The system in Fig. 3 can be instrumented as shown (for three fixed hypothesized delays) in Fig. 5. Fig. 5 also is called a time delay beam fomer. It is presumed that $s(t)$ is a sampled version of the original broadband time signal and that the sampling rate is large in comparison with the required Nyquist rate. In particular, the sampling

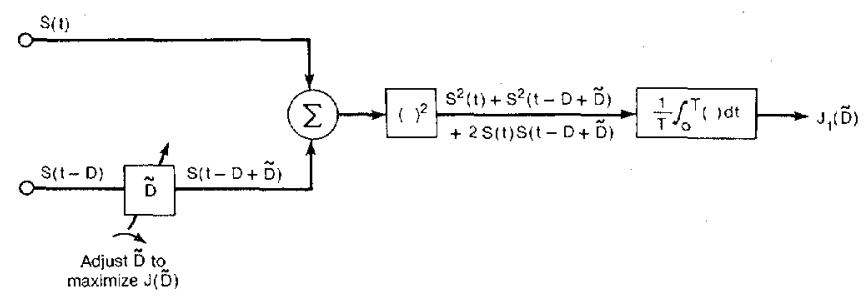

Fig. 3. Conceptual delay, sum, square and integrate configuration.

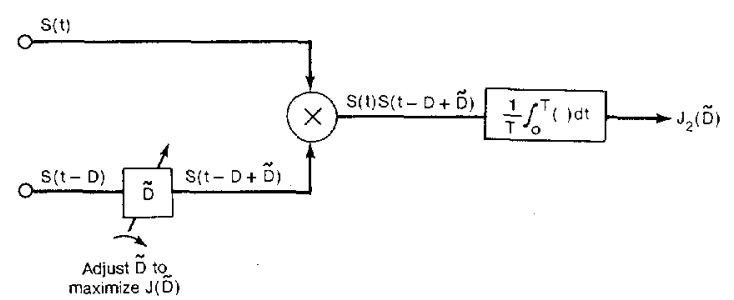

Fig. 4. Conceptual cross correlator configuration.

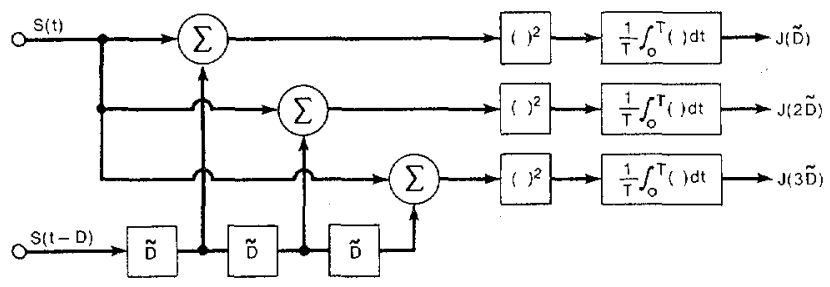

Fig. 5. Time-domain beam former instrumentation of one conceptual configuration for three hypothesized delays.

rate is much greater than twice the bandwidth. (A further discussion of beam formers can be found in Pridham and Mucci [47] and D'Assumpcao [16].) An example of applying the configuration of Fig. 5 to a pulse signal is shown in Fig. 6. Note that in the example, Fig. 6 , when the hypothesized time delay equals the true time delay, the system output power achieves a maximum. The other system configuration, shown in Fig. 4, including prefilters can be instrumented by a generalized crosscorrelation (GCC) function. This function can be estimated using the Fortran digital computer program in Programs for Digital Signal Processing, edited by the DSP committee [17]. Briefly, it consists of taking the inverse fast Fourier transform (FFT) of the product of a weighting function and the estimated complex cross-power spectrum.

\section{Attaining the Cramer-Rao Lower Bound By a GCC FUNCTION}

The paper on generalized cross correlators by Knapp and Carter [33] puts in perspective several suggested methods of filtering and cross correlation for time delay estimation including the often useful smoothed coherence transform (SCOT) or "rehocence" method that prewhitens and then cross correlates. (See, for example, the paper by Kostic [32] and references contained therein.) Recent extensions to that work, including simulation experiments, have been reported by Hassab and Boucher [27], and detection performance of the SCOT by Kuhn [35]. In related work Chan, Hattin, and Plant [13], using the results of Hamon and Hannan [25], give the minimum variance of time delay error in terms of the number of time samples, the number of frequency points to average, 

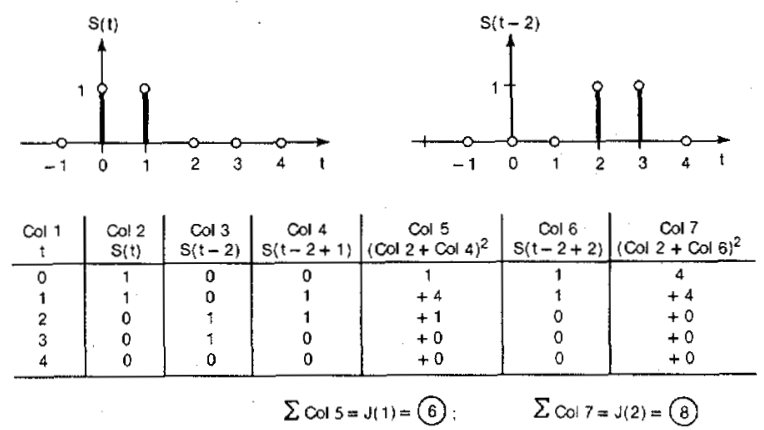

Fig. 6. Example of pulsed arrival for one instrumentation.

and a dimensionless SNR-type term. That work is consistent with the results of Carter [8], that the Cramer-Rao lower bound (CRLB) for the variance of time delay errors for the physical problem modeled by (1) is given by

$$
\sigma^{2}(\hat{D}-D) \geqslant \frac{2 \pi}{\left\{2 T \int_{0}^{\omega H} \frac{C(\omega)}{[1-C(\omega)]} \omega^{2} d \omega\right\}}
$$

where

1) $\omega_{H}$ is the highest source frequency after propagation to the receiver,

2) $T$ is the observation time, and

3) $C(\omega)$ is the magnitude-squared coherence (MSC). Equation (3a) can be expressed in terms of SNR by observing that the MSC is defined by

$$
C(\omega)=\frac{\left|G_{r_{1} r_{2}}(\omega)\right|^{2}}{\left[G_{r_{1} r_{1}}(\omega) G_{r_{2} r_{2}}(\omega)\right]} \leqslant 1, \quad \forall \omega .
$$

The MSC in (3b) for the model in (1) gives

$$
C(\omega)=\frac{G_{s s}^{2}(\omega)}{\left\{\left[G_{s s}(\omega)+G_{n_{1} n_{1}}(\omega)\right]\left[G_{s s}(\omega)+G_{n_{2} n_{2}}(\omega)\right]\right\}}
$$

where $G(\omega)$ is the auto- or cross-power spectrum at frequency $\omega$ of the subscripted random process(es). Equation (3a) agrees with estimated variances for selected cases of a digital simulation by Scarbrough, Ahmed, and Carter [50]. Utilizing (3c), (3a) is plotted as a function of center frequency and SNR in decibels (10 $\log _{10}$ of power) by Quazi [49] for the case of SNR independent of frequency in the signal band.

Several clarifying facts should be pointed out about (3). First, it is dimensionally correct. Second, in the underwater acoustics problem it is an implicit function of range; this is because signal power at the receiver is a function of propagation loss (conceptually owing to effects like spreading loss), which itself is a (or can be an extremely complicated) function of range. Third, the CRLB given by (3a) presumes that the signal and noise power spectral densities are known and that prior to cross correlation the received waveforms $r_{1}(t)$ and $r_{2}(t)$ in (1) are filtered by filters with transfer functions $H_{1}(f)$ and $H_{2}(f)$, respectively; the filters both have the same phase and the characteristic that the product of $H_{1}(f)$ with the complex conjugation of $H_{2}(f)$ forms the real weighting function given by

$$
W(f)=H_{1}(f) H_{2}^{*}(f)=C\left(f^{\prime}\right) /\left\{\left|G_{r_{1} r_{2}}(f)\right|[1-C(f)]\right\} .
$$

Equation (4) agrees with the equation between (17) and (18) of Hannan and Thomson [26]; following proper manipulation, (4) agrees with (17) of Hahn [23]. See Carter [8] for the specific filter characteristics for the case of more than two filters. (In the interest of completeness, we note that there is a typographical error in the filter transfer function given by (5) in Hahn [23].) In order to estimate the GCC function the estimated (complex) cross-power spectrum between the two received signals is multiplied by a weighting function $W(f)$, and then the inverse discrete Fourier transform (DFT) is computed via digital signal processing techniques such as the FFT. (As noted by Knapp and Carter [33], since the variance of the phase error is inversely proportional to MSC over one minus MSC, the GCC function with the ML weighting of (4) attaches most weight to the estimated phase when the variance of the estimated phase error is lowest. The work by Cleveland and Parzen [15] is one of the early references to using phase to determine time delay.) Another reference to using phase is [13]. A fourth item that must be considered in practice when using (3) is that often $W(f)$ is a function of unknown spectral quantities that must be estimated; in this case, the process of time delay estimation involves estimating the complex cross-power spectrum and the coherence function. The derivation of (3) presumes the spectral quantities needed for $W(f)$ are known. Finally (3) presumes Gaussian received signals. When the source radiates a sinusoidal signal, a tighter Barankin bound (poorer performance) occurs at low SNR. A more complete discussion is given in this issue by Chow and Schultheiss [14].

\section{Standard Deviation of Bearing Error}

The trigonometric relationship between time delay and bearing yields the result (see, e.g., Tacconi [60]) that the standard deviation of the bearing error in radians is given by

$$
\sigma(\hat{B}-B)=\sigma(\hat{D}-D) C /(L \sin B)
$$

where

1) $B$ is the bearing in radians;

2) $L$ is the distance between the two sensors (or subarrays); and

3) $C$ (without a frequency argument) is the speed of sound in water.

This result does not take into account uncertainty in sensor position which increases the variance by an additive amount and will be discussed later. (Note that (5) is dimensionally correct.) Substituting (3) into (5) gives the two-hydrophone result of MacDonald and Schultheiss [37]. Equations (3) and (5) show the effects of array length, source bearing, observation time, and coherence on bearing accuracy. It is noteworthy that when the pairwise sensor coherence is low we can still theoretically attain good performance if the source bandwidth is large or if we cluster a number of sensors together and beam form to increase the observed (or output) SNR. Extensions to (5) for endfire approximations are given by Hinich [29]

It should be noted that these results [(3) and (5)] pertain to a stationary processes large observation time (the so-called SPLOT condition; see, e.g., Van Trees [62, Parts I and III]) of one member function of a nominally broad-band Gaussian 
random process observed in a nondispersive medium with sensors at known positions and with known SNR. Thus, in practical applications, these results will serve as a bound on performance and not necessarily an absolute indicator of performance. Work with non-Gaussian signals is discussed by Hilliard and Pinkos [28]. When the noises are correlated, the results are more complicated; this problem has been treated by Howell [30] and Bangs [4]. For dispersive medium effects, see Kirlin [31]. Correlator configurations that both detect and estimate bearing for narrow-band signals are considered by Miller [40]. The problem of processes with random relative phase is treated by Wax [63].

The problem of estimating time delay between two received signals in noise is closely related to the work by Hahn [20] and Hahn and Tretter [22]. In that work, the problem is to estimate the $M-1$ components of the relative time delay parameter vector $\left(D_{2}-D_{1}, D_{3}-D_{1}, \cdots, D_{M}-D_{1}\right)$ from the $M$ received waveforms,

$$
r_{i}(t)=s\left(t-D_{i}\right)+n_{i}(t), \quad i=1, M .
$$

Other work related to the reduction in pairwise time delay error due to additional sensors has been reported by Schultheiss [54] and Bjфrn $\phi$ [7]. For passive localization in a plane, three unique sensors (or subarray beam former outputs) are required. In general, we define the (relative) time delay as

$$
D_{i j}=D_{i}-D_{j}, \quad i, j=1,2,3 \text {. }
$$

By knowing (or estimating) the relative delays $D_{21}=D_{2}-D_{1}$ and $D_{32}=D_{3}-D_{2}$ and the sensor positions, we can estimate the position of the acoustic source. In particular, we find the position in terms of polar coordinates, i.e., range $R$ and bearing $B$ from the central sensor according to the exact relationship (see, e.g., Griffiths, Stocklin, and Van Schooneveld [19], Carter [12] , and references contained therein)

$$
R=\frac{\left\{L_{1}\left[1-\left(\frac{C D_{21}}{L_{1}}\right)^{2}\right]+L_{2}\left[1-\left(\frac{C D_{32}}{L_{2}}\right)^{2}\right]\right\}}{\left[2\left(\frac{C D_{32}}{L_{2}}-\frac{C D_{21}}{L_{1}}\right)\right]}
$$

and an expression for bearing is

$$
B=\cos ^{-1}\left(\frac{\left[L_{2}^{2}-2 R_{2} C D_{32}-\left(C D_{32}\right)^{2}\right]}{\left[2 R_{2} L_{2}\right]}\right)
$$

where $L_{1}$ and $L_{2}$ are the distances between sensor pairs depicted in Fig. 7. For large $R$ the middle term in the arcosine expression, (8), becomes dominant, consistent with (2).

\section{VARIANCE OF RANGe ERROR}

The variance of the range error has been shown by Hahn [21] to be dominated by the errors in estimating the difference in time delays. In particular, the variance of the range errors is

$$
\sigma^{2}(\hat{R}-R)=C^{2} \sigma^{2}\left[\hat{D}_{32}-\hat{D}_{21}-\left(D_{32}-D_{21}\right)\right]\left(R / L_{e}\right)^{4}
$$

where

1) $R$ is the range;

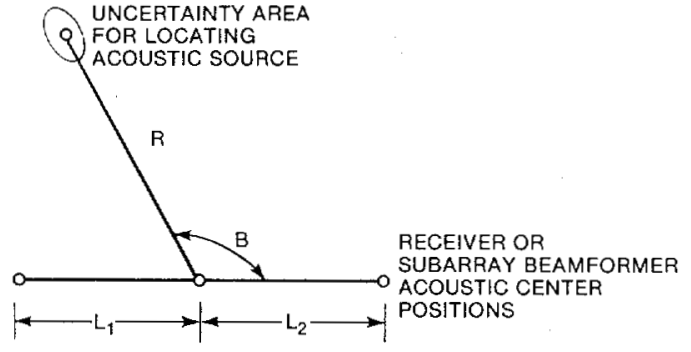

Fig. 7. Pictorial definition of range $R$, bearing $B$ and subarray separations $L_{1}$ and $L_{2}$.

2) $L_{e}$ is the effective half array length (i.e., the sine of the bearing times the interarray separation);

3) $C$ is the speed of sound in water; and

4) $\sigma^{2}(\mathrm{C})$ is the variance of the error in time delay difference measurements.

When comparing (9) with other work, it is noteworthy that by rewriting that equation in terms of the total array length, it apparently becomes 16 times larger. Using Carter [9] we readily can show that for the optimum range and bearing sonar signal processor the variance of the range error is given by

$$
\sigma^{2}(\hat{R}-R)=12 \sigma^{2}(\hat{B}-B) R^{4} / L_{e}^{2}
$$

where the bearing errors are in radians. Using (5) to relate time delay errors to bearing errors and observing that for an equispaced three-sensor array we have twice the array length and 1.5 times as many sensors as used to obtain (5), we postulate that the variance of bearing errors can be approximated by

$$
\sigma^{2}(\hat{B}-B)=C^{2} \sigma^{2}\left(\hat{D}_{31}-D_{31}\right)(2 / 3)\left(1 /\left[2 L_{e}\right]\right)^{2}
$$

where $\sigma^{2}()$ is the variance of the error when estimating the time delay from sensor 3 to 1 . We note that (11) depends on the effective array length but not on the range to the source $R$. Substituting (11) into (10) yields

$$
\sigma^{2}(\hat{R}-R)=2 C^{2} \sigma^{2}\left(\hat{D}_{31}-D_{31}\right)\left(R / L_{e}\right)^{4} .
$$

The variance of the error in estimating the difference of time delays (used in the denominator of (8), the ranging equation) is approximately twice as great as the variance of the error in estimating $D_{31}$. (With regard to the approximation see Hahn [23] for an evaluation of the covariance of the errors in estimating $D_{21}$ and the errors in estimating $D_{31}$.) Therefore, we conclude that (12) and (9) agree. Comparing (12) with (11) note that the range error variance and bearing error variance both depend linearly on time delay error variance; however, unlike bearing errors ranging errors are highly dependent on the ratio of the true source range to the length of the effective baseline. Indeed, in passive ranging, it is the fourth power of the range relative to the effective array length that is important to the range error variance. This mathematical result helps develop physical insight into the difficulties of estimating the position of an acoustic source. Specifically, we see that inherent in the physics of the passive ranging problem is the need to have long baselines, extremely accurate time delay estimates, or small (true) range from the receiving array to the acoustic source, or all three. In addition, we point out here that owing to propagation loss the SNR or coherence at the receivers is a function of range from the source to the receivers. Hence, for 
certain underwater acoustic applications, (12) is a stronger function of range than might otherwise be explicitly apparent.

An empirical method for estimating the variance necessary in (12) was found by Schneider [52] of ENSCO, Inc., who observed from (7) that

$$
D_{21}+D_{32}-D_{31}=0 \text {. }
$$

That is, in the absence of estimation noise independent of source and receiver geometries, the time it takes an acoustic wave to travel from sensor 3 to 2 plus the travel time from sensor 2 to 1 should equal the travel time from sensor 3 to 1 . Hence, a useful internal consistency check for the estimated time delay parameters is $\hat{D}_{21}+\hat{D}_{32}-\hat{D}_{31}$. The mean-square value of this three-term sum represents a practical guide to performance bounds without consideration for source and receiver motion. Schneider [52] and Beam and Carter [5] have found this guide useful for actual sea data. An extension to a generalized broadband Doppler or relative time compression (RTC) internal consistency check for moving sources has also been done by Schneider [51].

\section{Performance Bounds Found by Hypothetical ARRAYS}

In the work of MacDonald and Schultheiss [37] the optimum processor for bearing estimation was derived assuming planewaves, Gaussian signals and uncorrelated Gaussian noises of equal strength. The performance of such a system was found together with a bound obtained by considering a hypothetical system in which half the elements are placed at each end of the available aperture. (In practice, though, we postulate that we would require half-wavelength spacing at the design frequency for each subarray; see, e.g., Fig. 8.) An extension (in concert with Pryor [48]) to unequal SNR was reported by Carter [10] . These results showed that at low unequal SNR the available hydrophones should be divided into two equal groups. Thus we see that passive sonar systems designed to be optimum bearing estimators for equal SNR are also optimum for unequal SNR in the important case when the SNR is low.

When the acoustic source is sufficiently close to the center of the receiving array relative to the length of the array, then, unlike Fig. 8 , the received wavefronts appear curved as shown in Fig. 9. A bound on the best ranging performance can be calculated by distributing a quarter of the receiving elements at each end and the remaining half of the elements at the middle of the array aperture (see Carter [9]). We emphasize that the bound is good even though the array configuration is theoretical in nature. These results hold for known sensor positions, and extensions are underway to the moving sensor. In particular, Schultheiss and Ianniello [56] consider an ensemble of statically deformed arrays and correctly infer that an equispaced line array might in some situations be preferable to three clustered subarrays. Work on a moving array is in progress. When the source uncertainty area is to be minimized and sensor positions are known, a bound on performance is calculated by a sensor distribution that places one third of the available sensing hydrophones at each end and the middle of the available aperture [9]. Of course, if sensor positions are not known exactly, performance is also expected to be worse than the performance bound found when sensor position is known.

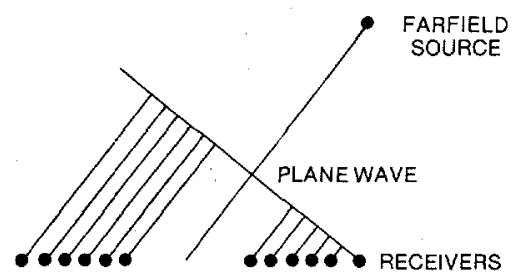

Fig. 8. Plane wave model for bearing estimation.

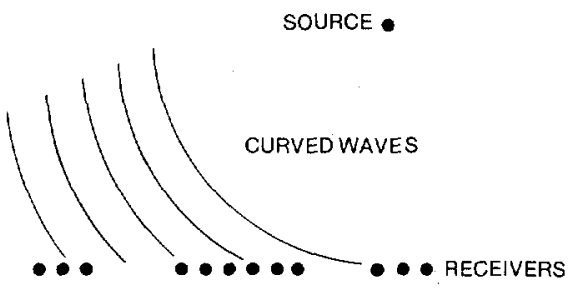

Fig. 9. Curved wave model for range estimation.

\section{ViII. Configuration for Optimum Position ESTIMATION}

Configurations for the optimum processor for range and bearing estimation are discussed by Hahn [23] and Carter [9] . One configuration presented by Carter [9] is shown in Fig. 10. We refer to this configuration as a focused beam former because it constrains the hypothesized time delays to focus the acoustic energy from a hypothesized position. It effectively presumes that the receiving sensor positions are known. Unlike the focused beam former, the ML estimate for the time delay vector maximizes the output power $J$ of this network and does not constrain the power to be focused at one hypothesized range $R$ and bearing $B$. A second realization is to form all possible GCC pairs and combine the delay estimates from each pair according to the rules of Hahn [23]. The input signals are filtered with particular signal-enhancing filters, all with the same phase (e.g., symmetric digital finite impulse response (FIR) filters, all with the same bulk or group time delay); and the delayed outputs are summed, squared, and averaged. The particular filter transfer functions needed to yield the best performance are given by Carter [8]. The ML range and bearing estimates are achieved by adjusting hypothesized range and bearing parameters that, in turn, give rise (with knowledge of the receiving hydrophone positions) to the appropriate time delays to be inserted after filtering. When the total system output power $J$ is maximized, the selected hypothesized range and bearing estimates are the ML source position estimates. In the limit of large observation time these ML estimators are the minimum variance source-position estimators.

For systems with the available elements grouped into three subarrays, a suboptimum realization is shown in Fig. 11; it can be achieved at lower cost and is postulated to have performance similar to that of the optimum system in Fig. 10. In the Fig. 11 suboptimum system we first beam form each of the three subarrays. Subsequently, we perform a GCC on the forward and middle and the middle and aft beam former outputs and read off the correlator abscissa showing the peak. Many commercially available correlators perform similar functions. We also can form a GCC on the forward and after beam former output and form the internal consistency check. For purposes of analysis, we model the GCC as prefilters, a variable delay, 


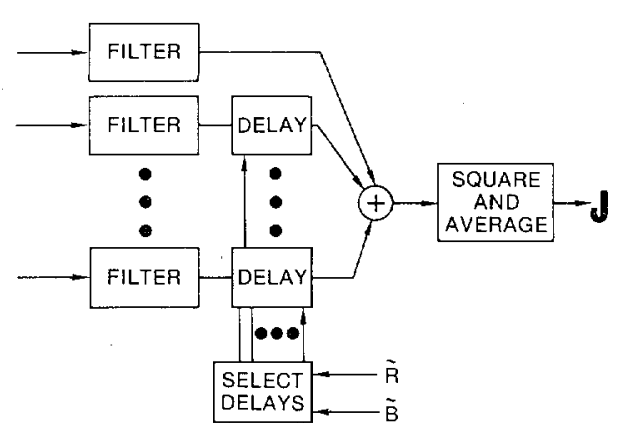

Fig. 10. Focused beam former for range and bearing estimation.

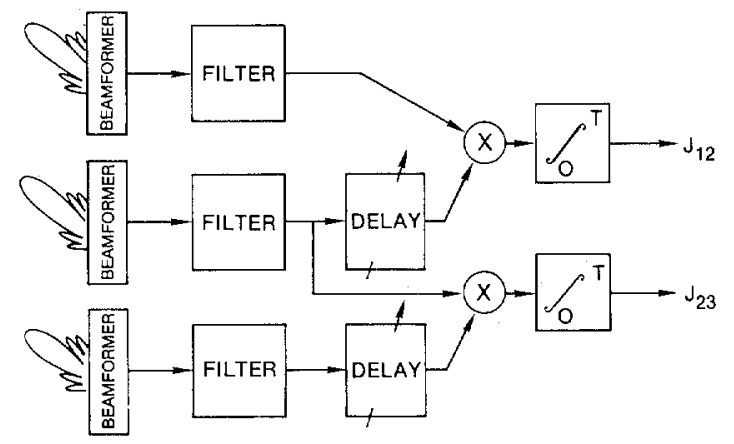

Fig. 11. Suboptimum realization of focused beam former.

and $T$-second integrator. Then the delay estimates found by the Fig. 11 realization are inserted in the ranging equation (8) to obtain a range estimate.

\section{Other Limitations to Performance}

In practice, both the optimum and suboptimum realizations are degraded by realistic factors. A most significant factor can be that signal processing implementations derived from treating the planar problem may not suit even the simple multipath model shown earlier in Fig. 1. These and other significant environmental factors, such as the sound speed profile, the bottom depth, slope, and layering together with bottom loss, often need to be considered. Limitations due to source motion have been studied and reported by Gerlach [18]. These types of studies provide information on the fundamental bounds on performance.

Two other potentially significant effects on passive sonar localization are uncertainty in sensor position and failure to compensate for broad-band Doppler or RTC. The first cause of error to be discussed is depicted in Fig. 12 and has been studied and reported by Carter [11]. As mentioned earlier, additional work has been conducted and is continuing by Ianniello and Schultheiss. In bearing estimation two receivers of known position are needed. Consider the two left receivers indicated by squares for the actual positions. If the receiver were perturbed by an amount $P$ (small in comparison with the sensor separation $L$ ) from the position of the square in Fig. 12 to the solid dot position, then the bearing estimate would be in error by approximately an amount of $P / L$ radians. Of course, if $P$ were a random perturbation, the variance of the bearing error could be reduced by averaging a series of independent bearing measurements over a very large observation time. The total bearing error then consists of the sum of two terms, one having

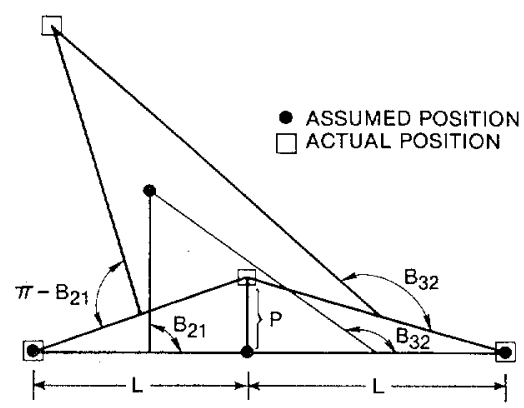

Fig. 12. Effect of receiver positional uncertainty on localization.

to do with SNR and the other having to do with array perturbation. Fundamentally, the relative ranging errors due to unknown random sensor perturbation $P$ about some known mean $P$ are approximately given by

$$
\sigma(\hat{R}-R) / R \cong(\sigma(\hat{P}-P) / L)\left(2 R / L_{e}\right)
$$

where

1) $\sigma(\hat{P}-P)$ is the standard deviation of the middle sensor perturbation from a line connecting the two end sensors;

2) $R$ is the range to the source;

3) $L_{e}$ is the effective half array length; and

4) $L$ is half the total array length.

Thus, the ratio of the perturbations relative to the array length and the range relative to the effective array length are the dominant contributors to this type of relative ranging error. We note that array perturbations can be viewed as time delay errors with a simple trigonometric mapping for small perturbations. Not unexpectedly then, the form of (14) is consistent with (9).

\section{Velocity Estimation}

Another important type of degradation is caused by failure to compensate for the "deterministic" part of source motion. A simple but useful model of received signals is discussed by Abraham and Carter [1]. A more complete discussion of the model and the ML estimator for the fundamental motion parameter, tantamount to time delay rate or bearing rate, is given by Knapp and Carter [34]. Theoretically though, a suboptimum realization is depicted in Fig. 13. Work in this area has been done at the Naval Ocean Systems Center (NOSC) by Stradling [58], McCarthy [38], Mohnkern [41], and Trueblood [61]. Other models include one discussed by Adams, Kuhn, and Whyland [2], Schultheiss [53], Schultheiss and Weinstein [55], and Stein [57]. They treat the moving source problem by considering a truncated Taylor series expansion. Such models physically include time delay, time delay rate, and time delay acceleration. Related work includes an extended MSC estimator, similar to an algorithm by Trueblood [61], that was reported by Patzewitsch, Srinath, and Black [46]. Other related work on tracking of moving time delays for velocity estimation has been reported by Meyr [39] and Lindsey and Meyr [36]. Work in this area, including sensing systems for determining train speed, is being continued by Meyr and his students. The work by Moura [43] and Moura and Baggeroer [42] also is concerned with passive velocity estimation. For many practical 


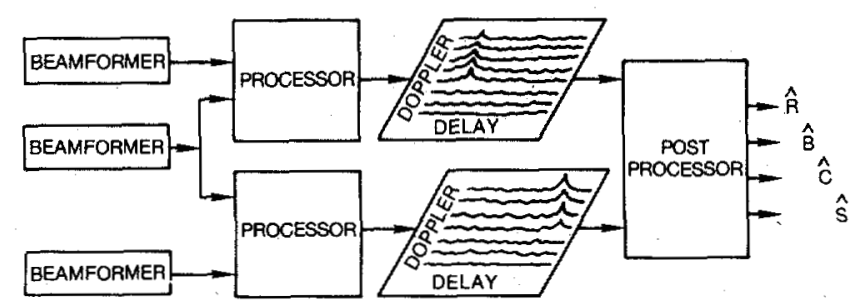

Fig. 13. Theoretical procedure for range, bearing, course and speed estimation.

geometries when the source range is long compared with the receiver baseline and the SNR is large so that $T$ can be kept small, the estimation of RTC or generalized Doppler is not necessary in order to estimate time delay. For other geometries, RTC or time delay rate estimation is necessary. Even when it is not necessary, RTC estimation can provide rapid early indications of source velocity.

It is analytically useful in this estimation procedure to decouple velocity into two orthogonal components: bearing rate or cross line-of-sight speed (CLSS) and range rate or radial lineof-sight speed (RLSS). In particular, the CLSS is defined as the projection of the velocity vector on the perpendicular to the bearing line, and the RLSS is the projection on the bearing line. The problem is to determine the accuracy with which source velocity can be determined. Theoretical considerations indicate that CLSS is much easier to estimate than RLSS. The RTC can be estimated using Knapp and Carter [34] ; see also Stein [57]. Bearing can be estimated using MacDonald and Schultheiss [37], and range can be estimated by means of the triangulation methods of Hall and Hayford [24] and Hilliard and Pinkos [28].

From these results Carter [12] has shown the variance in CLSS errors is dominated by range relative to the baseline projection and the variance of the RTC estimation error.

Further, the standard deviation of the CLSS errors is predominantly a function only of the range relative to the effective baseline steered at the source and the standard deviation of the RTC errors. In most practical ways, it is not a function of the course or speed of the source or errors in time delay. However, when the coherence or SNR is low and the observation time $T$ is short, errors in both time delay and RTC or CLSS will be large. Theriault and Berkman [59] have derived an approximate expression for the standard deviation of the errors in the radial component of velocity.

RLSS errors, like CLSS errors, are not predominantly a function of the course or speed of the source. However, unlike CLSS errors, RLSS errors depend on the square of the range relative to the effective baseline.

It was corroborated by computer simulation that the CLSS is much easier to estimate than the RLSS. Also, doubling the range-to-effective-baseline ratio doubled the CLSS errors and quadrupled the RLSS errors. Further, the errors in CLSS and RLSS did not appear to depend on either time delay errors explicitly or source velocity.

\section{Summary}

Time delay estimation is an important part of passive sonar signal processing. An overview of applied research in passive sonar signal processing estimation techniques has been presented. One problem that motivates time delay estimation is the estimation of the position and velocity of a moving acoustic source. A discussion of this problem in terms of estimating time delay has been presented. In order to develop an understanding of the signal processing required, an approach of decoupling the problem into multipath and planar components was followed. Optimum estimators for acoustic source position were presented for the planar problem and related to the optimum time delay vector estimator with a focusing constraint. In particular, the focused beam former with appropriate prefilters is, in some sense, the best range and bearing position estimator.

\section{REFERENCES}

[1] P. B. Abraham and G. C. Carter, private communications, 1978; see also G. C. Carter and P. B. Abraham, "Estimation of source motion from time delay and time compression measurements," J. Acoust. Soc. Amer., vol. 67, no. 3, pp. 830-832, 1980.

[2] W. B. Adams, J. P. Kuhn, and W. P. Whyland, "Correlator compensation requirements for passive time delay estimation with moving source or receivers," IEEE Trans. Acoust., Speech, Signal Processing, vol. ASSP-28, no. 2, pp. 158-168, 1980.

[3] R. A. Altes, "Target position estimation in radar and sonar, and generalized ambiguity analysis for maximum likelihood parameter estimation," Proc. IEEE, vol. 67, no. 6, pp. 920-930, 1979.

[4] W. J. Bangs, II, "Array processing with generalized beamformers," Ph.D. dissertation, Yale Univ., New Haven, CT, 1971; see also the work by Bangs and Schultheiss appearing in Griffiths, Stocklin, and Van Schooneveld, 1973.

[5] J. P. Beam and G. C. Carter, private communication on passive localization sea test results, 1980.

[6] J. S. Bendat and A. G. Piersol, Engineering Applications of Correlation and Spectral Analysis. New York: Wiley, 1980.

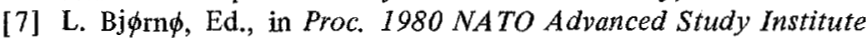
on Underwater Acoustics and Signal Processing. Boston, MA: Reidel, 1981.

[8] G. C. Carter, "Time delay estimation," Ph.D. dissertation, Univ. Connecticut, Storrs, 1976 (also available as NUSC TR 5335 through NTIS under AD A025408).

[9] _- "Variance bounds for passively locating an acoustic source with a symmetric line array," J. Acoust. Soc. Amer, vol. 62, no. 4, pp. 922-926, 1977.

[10] _ "Optimum element placement for passive bearing estimation in unequal signal-to-noise ratio environments," IEEE Trans. Acoust., Speech, Signal Processing, vol. ASSP-26, no. 4, pp. 365$366,1978$.

[11] — "Passive ranging errors due to receiving hydrophone position uncertainty," J. Acoust. Soc. Amer., vol. 65, no. 2, pp. 528-530, 1979.

[12] - "Sonar signal processing for source state estimation," in EASCON'79 Rec., IEEE Pub. 79CH 1476-1-AES, 1979.

[13] Y. T. Chan, R. V. Hattin, and J. B. Plant, "The least squares estimation of time delay and its use in signal detection," IEEE Trans. A coust., Speech, Signal Processing, vol. ASSP-26, no. 3, pp. $217-$ 222, 1978; see also Y. T. Chan, J. M. Riley, and J. B. Plant, "A parameter estimation approach to time delay estimation and signal detection," IEEE Trans. Acoust, Speech, Signal Processing, vol. ASSP-28, no. 1, pp. 8-16, 1980.

[14] S. K. Chow and P. M. Schultheiss, "Delay estimation using narrowband processes," this issue, pp. 478-484.

[15] W. S. Cleveland and E. Parzen, "The estimation of coherence, frequency response and envelope delay," Technometrics, vol. 17, no. 2, pp. 167-172, 1975.

[16] H. A. D'Assumpcao, "Some new signal processors for arrays of sensors," IEEE Trans. Inform. Theory, vol. IT-26, no. 4, pp. 441-453, 1980.

[17] Digital Signal Processing Committee, Ed., Programs for Digital Signal Processing. New York: IEEE Press (dist. Wiley), 1979.

[18] A. A. Gerlach, "Acoustic transfer function of the ocean for a motional source," IEEE Trans. Acoust., Speech, Signal Processing, vol. ASSP-26, no. 6, pp. 493-501, 1978. 
[19] J. W. R. Griffiths, P. L. Stocklin, and C. Van Schooneveld, Eds., Signal Processing. New York: Academic, 1973 (including P. Heimdal and F. Bryn, "Passive ranging techniques"; E. B. Lunde, "Wavefront stability in the ocean"; W. J. Bangs and P. M. Schultheiss, "Space-time processing for optimal parameter estimation").

[20] W. R. Hahn, "Optimum estimation of a delay vector caused by a random field propagating across an array of noisy sensors," Ph.D. dissertation, Univ. Maryland, College Park, 1972 (available as NOL TR 72-120 through NTIS AD 751555).

[21] - private communication on passive ranging, 1973.

[22] W. R. Hahn and S. A. Tretter, "Optimum processing for delayvector estimation in passive signal arrays," IEEE Trans. Inform. Theory, vol. IT-19, no. 5, pp. 608-614, 1973.

[23] W. R. Hahn, "Optimum signal processing for passive sonar range and bearing estimation," J. Acoust. Soc. Amer., vol. 58, no. 1 , pp. 201-207, 1975.

[24] J. B. Hall and R. I. Hayford, private communications on certain processing techniques, 1977 .

[25] B. V. Hamon and E. J. Hannan, "Spectral estimation of time delay for dispersive and non-dispersive systems," Appl. Statist., vol. 23 , no. 2, pp. 134-142, 1974.

[26] E. J. Hannan and P. J. Thomson, "Estimating group delay," Biometrika, vol. 60 , no. 2 , pp. 241-253, 1973; see also E. J. Hannan and P. J. Thomson, "Delay estimation," this issue, pp. 485-490.

[27] J. C. Hassab and R. E. Boucher, "A quantitative study of optimum and suboptimum filters in the generalized correlator," in IEEE 1979 Int. Conf. Acoust., Speech, Signal Processing Rec., 79CH1379-7 ASSP, pp. 124-127, see also J. C. Hassab and R. E. Boucher, "Performance of the generalized cross correlator in the presence of a strong spectral peak in the signal," this issue, pp. 549-555.

[28] E. J. Hilliard, Jr. and R. F. Pinkos, "An analysis of triangulation ranging using beta density angular errors," J. Acoust. Soc. Amer. vol. 65 , pp. 1218-1228, May 1979.

[29] M. J. Hinich, "Estimating bearing when the source is endfire to an array," J. Acoust. Soc. Amer., vol. 65, no. 3, pp. 845-846, 1979.

[30] L. R. Howell, "Passive sonar bearing estimation in the presence of highly anisotropic noise fields," Ph.D. dissertation, Catholic Univ. America, Washington, DC, 1978.

[31] R. L. Kirlin, "Augmenting the maximum likelihood delay estimator to give maximum likelihood direction," IEEE Trans. Acoust., Speech, Signal Processing, vol. ASSP-26, no. 1, pp. 107108,1978

[32] L. Kostić, "Local steam transit time estimation in a boiling water reactor," this issue, pp. 555-560.

[33] C. H. Knapp and G. C. Carter, "The generalized correlation method for estimation of time delay," IEEE Trans. Acoust., Speech, Signal Processing, vol. ASSP-24, no. 4., pp. 320-327, i976.

[34] - "Estimation of time delay in the presence of source or receiver motion," J. Acoust. Soc. Amer., vol. 61, no. 6, pp. 1545$1549,1977$.

[35] J. P. Kuhn, "Detection performance of the smooth coherence transform," in 1978 IEEE Int. Conf. A coust., Speech, Signal Processing Rec., 78CH1285-6ASSP, pp. 678-683.

[36] W. C. Lindsey and H. Meyr, "Complete statistical description of the phase-error process generated by correlative tracking systems," IEEE Trans. Inform. Theory, vol. IT-23, no. 2, pp. 194-202, 1977.

[37] V. H. MacDonald and P. M. Schultheiss, "Optimum passive bearing estimation in a spatially incoherent noise environment," $J$. Acoust. Soc. Amer., vol. 46, pp. 37-43, 1969.

[38] S. J. McCarthy, "Likelihood filtering of passive ambiguity surfaces," Naval Ocean System Center, San Diego, CA, Tech. Rep. $408,1979$.

[39] H. Meyr, "Delay lock tracking of stochastic signals," IEEE Trans. Commun, vol. COM-24, no. 3, pp. 331-339, 1976.
[40] L. E. Miller, "Capabilities of multiplicative array processing as signal detector and bearing estimator," Ph.D. dissertation, Catholic Univ. America, Washington, DC, 1974 (NTIS AD A004-587).

[41] G. Mohnkern, private communication, 1975.

[42] J. M. F. Moura and A. G. Baggeroer, "Passive systems theory with narrow-band and linear constraints: Part I-Spatial diversity," IEEE J. Ocean Eng., vol. OE-3, pp. 5-13, Jan. 1978.

[43] J. M. F. Moura, "Passive systems theory with narrow-band and linear constraints: Part II-Temporal diversity," IEEE J. Ocean Eng., vol. OE-4, pp. 19-30, Jan. 1979.

[44] A. V. Oppenheim, Ed., Applications of Digital Signal Processing. Englewood Cliffs, NJ: Prentice-Hall, 1978 (especially ch. 6 on sonar signal processing by A. B. Baggeroer).

[45] N. L. Owsley, "Source location with an adaptively focused array," in Proc. IEEE Conf. Decision and Control; see also N. L. Owsley and G. R. Swope, "Time delay estimation in a sensor array," this issue, pp. 519-523, and paper by Owsley in Bjфrn $\phi$ [7].

[46] J. T. Patzewitsch, M. D. Srinath, and C. I. Black, "Nearfield performance of passive correlation processing sonars," J. Acoust. Soc. Amer., vol. 64, no. 5, pp. 1412-1423, 1978; see also J. T. Patzewitsch, M. D. Srinath, and C. I. Black, "Near field performance of passive coherence processing sonars," IEEE Trans. Acoust., Speech, Signal Processing. vol. ASSP-27, Part I of II, no. 2 , pp. 573-582, 1979.

[47] R. G. Pridham and R. A. Mucci, "Digital interpolation beamforming for low-pass and bandpass signals," Proc. IEEE, vol. 67, no. 6, pp. 904-919, 1977.

[48] C. N. Pryor, private communications, 1978

[49] A. H. Quazi, "An overview on time delay estimate in active and passive systems for target localizations," this issue, pp. 527-533.

[50] K. Scarbrough, N. Ahmed, and G. C. Carter, "On the simulation of a class of time delay estimation algorithms," this issue, pp. $534-540$

[51] S. M. Schneider, private communication on coherent interarray processing, 1978.

[52] - private communication, 1979.

[53] P. M. Schultheiss, private communication on estimation of Doppler shift, 1977.

[54] —, "Locating a passive source with array measurements: A summary of results," in 1979 IEEE Int. Conf. Acoust., Speech, Signal Processing Rec., 79CH1379-7ASSP, pp. 967-970.

[55] P. M. Schultheiss and E. Weinstein, "Source tracking using passive array data," this issue, pp. 600-607.

[56] P. M. Schultheiss and J. P. Ianniello, "Optimum range and bearing estimation with tandomly perturbed arrays," J. Acoust. Soc. Amer, vol. 68, no. 1, pp. 167-173, 1980.

[57] S. Stein, "Algorithms for ambiguity function processing," this issue, pp. 588-599.

[58] C. Stradling, private communication, 1975

[59] K. B. Theriault and E. F. Berkman, private communications, 1978.

[60] G. Tacconi, Ed., A spects of Signal I. Boston, MA: Reidel, 1977 (including G. C. Carter, "The role of coherence in time delay estimation," pp. 252-256).

[61] R. Trueblood, private communication, 1975

[62] H. L. Van Trees, Detection, Estimation and Modulation Theory New York: Wiley, 1968; see also Part III, 1971.

[63] M. Wax, "The estimation of time delay between two signals with random relative phase shift," this issue, pp. 497-501; see also related manuscript submitted in 1980 to IEEE Trans. Inform. Theory.

G. Clifford Carter (M'70-SM'78), for a photograph and biography, see this issue, p. 462. 